\title{
European Rail Transportation
}

\begin{abstract}
Liebe Leserin! Lieber Leser!
Seit dem vor fünf Jahren erschienenen e\&i-Themenheft zur Eisenbahnsicherungstechnik haben wir Ihnen über Weiterentwicklungen auf diesem Gebiet in einzelnen Beiträgen berichten dürfen. Das vorliegende Heft positioniert sich nun breiter, nimmt sowohl auf strategische Ausrichtungen des Verkehrsträgers Schiene bei der "European Rail Transportation“ als auch auf aktuelle Sicherheitsfragen im Bahnverkehr Bezug. Ausdrücklich hervorzuheben ist, dass heimisches Know-how zur Schienenverkehrstechnik mittlerweile weit über die Landesgrenzen hinaus bekannt und gefragt ist. Ähnliches gilt für die Straßentelematik, wie ein abrundender Blick auf die gelungene Einführung der elektronischen Autobahnmaut in Österreich zeigt.
\end{abstract}

\section{Schiene}

\subsection{Marktposition der Bahnen in Europa}

Verlagerungen des Passagier- und Güterverkehrs von der Schiene zu Straße, Luft- und Schifffahrt sind keineswegs neu. Mit der europäischen Initiative „European Rail Transport Management System" (ERTMS) steuert man diesem Trend entgegen, um mit voranschreitender Erweiterung der Europäischen Union die Position der Schiene - vor allem beim Güterverkehr zu stärken. Einleitend wird dies in der Arbeit von A. Veider „Energising the expansion of ERTMS" beschrieben und eine Bündelung der Aktivitäten wichtiger europäischer Bahnen mit der Transportautomatisierungsindustrie verlangt.

Der Beitrag von $\mathrm{H}$. Steindl „Signaltechnik im 21. Jahrhundert" stellt auf eindrucksvolle Weise dar, dass die Netzbetreiber nach einer raschen Migration der derzeitigen Signaltechnik zu einem interoperablen System des 21. Jahrhunderts („European Train Control System“ ETCS zusammen mit „Global System for Mobile communication - Rail" GSM-R, beides als Subsysteme zu ERTMS) den Eisenbahnverkehrsunternehmen unter anderem günstigere Trassenpreise gewähren können, was für die Bahn zu deutlichen Marktvorteilen bei den Verkehrsträgern Straße - Schiene - Luft- und Schifffahrt führt.

\subsection{Betriebssimulation}

Durch den stetigen Wandel und Fortschritt im Zusammenhang mit dem Wettbewerb der Verkehrsträger um den Transportmarkt werden zur effizienten Betriebsführung der Bahn mächtige, vernetzt ablaufende Simulations- und Planungswerkzeuge unerlässlich. Im Beitrag von Herrn Professor Dr. Ostermann et al. "Anwendungen der Betriebssimulation im Eisenbahnwesen" wird anhand praktischer Beispiele darauf und auf die damit einhergehenden neuen Herausforderungen des Datenmanagements näher eingegangen.

Die im Rahmen von ERTMS und ETCS europaweit standardisierten Fahrweg- und Zugsicherungssysteme sind auch die moderne Basis für sichere und zuverlässige Betriebsführung im Schienennetz. Dazu stellen $H$. Steinbrecher et al. „Aktuelle Aspekte der Eisenbahnbetriebsführung - das Betriebsführungssystem ALINET von Alcatel" als eine Plattform für optimalen Betrieb der Schieneninfrastruktur vor.

\subsection{Sicherheitskomponenten für Schieneninfrastruktur und Fahrzeuge}

Eine grundlegende Rolle für den grenzüberschreitenden interoperablen Bahnverkehr in ganz Europa kommt dem im Rahmen von ETCS standardisierten "Eurobalise-Subsystem" mit standardisierten Einrichtungen entlang von Schienenstrecken sowie auf Triebfahrzeugen zu. Im Aufsatz von R. Hornstein et al. „The Eurobalise Subsystem - a technical reflection" werden exemplarisch mittels Analysen von Modell- bzw. Prototypkonfigurationen nicht nur die wesentlichen Subsystemeigenschaften dargelegt, sondern auch die einschlägigen Standards kritisch hinterfragt und mögliche Problemlösungen diskutiert. Dabei werden unter anderem Sender-/Empfängerkonzepte entsprechend den hohen funktionalen und Sicherheitsanforderungen im Bahnbetrieb vorgestellt.

Anschließend wird die Komplexität der Sicherheitsproblematik in Fahrzeugen sowie die Praxislösung dieser

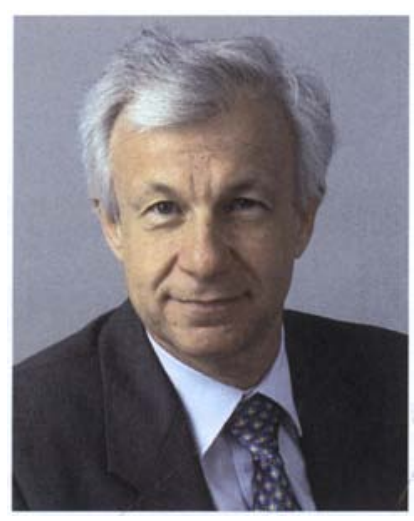

Dr. Helmut Malleck

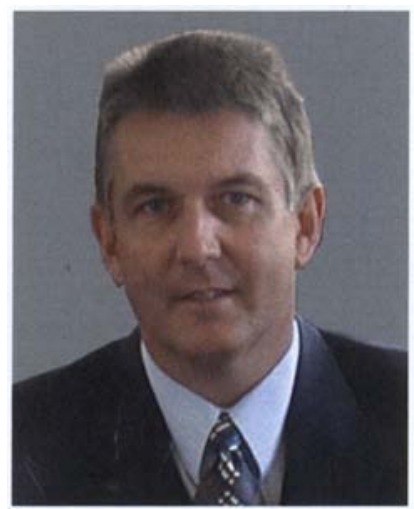

Dr. Alfred Veider Herausforderung von R. Eingang et al. im Beitrag „Sicherheit bei Schienenfahrzeugen am Beispiel der neuen E-Talent-Triebwagenreihe 4023/4024 der ÖBB" eindrucksvoll vor Augen geführt.

Fahrzeuge für den Betrieb ohne Fahrzeugführer weisen technische Besonderheiten auf, und es werden weitreichendere Anforderungen als an Züge für den konventionellen Betrieb gestellt. Die. Funktionsprinzipien werden anhand dreier Fahrzeugkomponenten von $\mathrm{H}$. Beismann und V. Schmidt in der Rubrik Praxis \& Wissen in dem Beitrag "Schienenfahrzeuge für den fahrerlosen U-Bahnbetrieb" am Beispiel RUBIN, dem ersten vollautomatischen U-Bahnwagen für Nürnberg, erläutert.

\section{Straße}

Abschließend soll in der Rubrik Praxis \& Wissen, wie eingangs erwähnt, noch ein Blick auf die Straße geworfen, der Bogen etwas weiter gespannt werden. Über das österreichische landesweit automatische elektronische Mautsystem berichtet K.E. Ambrosch in seinem Beitrag „Applied telematics - what makes the world's largest electronic tolling system work" in einer klaren technischen Darstellung, spannend und voll patriotischem Stolz. 
Wir wünschen Ihnen recht viel Vergnügen bei der Lektüre dieses Themenheftes!

Helmut Malleck

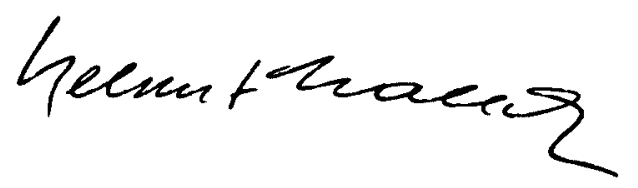

Alfred Veider

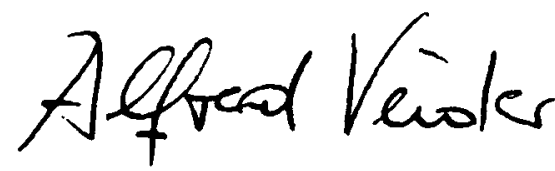

\section{Innovationspotenzial im Eisenbahnwesen Österreichs}

Europas bisher weitgehend nationale Eisenbahnen unterliegen seit geraumer Zeit einem der umfassendsten Veränderungsprozesse seit ihrem Bestehen. Die von der EU beabsichtigten Maßnahmen im Eisenbahnwesen, die durch verschiedene Richtlinien und die Pakete 2004 und 2006 definiert sind, erzwingen umfangreiche Reformen und das Einstellen auf einen nummehr ebenfalls liberalisierten Schienenverkehrsmarkt, auf dem in der Folge neue und unabhängige Eisenbahnverkehrsunternehmen (EVUs) erscheinen.

Damit soll schließlich erreicht werden, dass die Bahnen in der EU zu kräftigen Mitbewerbern zur Straße auf dem Verkehrsmarkt auftreten können. Dies zwingt $z u$ intensivem Handeln, das Änderungen im inhaltlichen, strategischen und damit auch im technologischen Bereich erfordert. führt:

Zur Verdeutlichung seien nur einige der EU-Vorgaben ange-

- Trennung von Absatz (Personen- und Güterverkehr) und Infrastruktur mit Verbot der Querfinanzierung,

- Liberalisierung des Netzzugangs (Förderung des Wettbewerbs),

- einheitliche technische Vorschriften für Hochgeschwindigkeitsverkehr und konventionelle Verkehre (Schaffung europaweit einheitlicher Systemmodule und technischer Parameter),

- und letztlich die Version eines einheitlichen Eisenbahnsystems für Europa in der Gruppierung „European Rail Research Advisory Council“" (ERRAC).

Vorstehende Gegebenheiten sind für die Eisenbahnverkehrsunternehmen, die einschlägige Industrie und nicht zuletzt auch für die wissenschaftlichen Institutionen eine große Herausforderung. Für den Standort Österreich und das in diesem Lande erworbene Know-how ist es unabdingbar, F \& E-Aktivitäten zu bündeln, um auch in Zukunft den derzeit noch gehaltenen Vorsprung der österreichischen Eisenbahnindustrie abzusichern, weiter auszubauen und damit auch breiter zu verankern.

Diesem Ziel dienen Aufbau und Etablierung des Ende 2003 gegründeten „Rail Technology Cluster Austria“ (RTCA), in dem sich Bahn-affine Industrie von Großunternehmen bis hin zu Klein- und Mittelbetrieben (KMUs), wissenschaftliche Institutio- nen und Eisenbahnverkehrsunternehmen zur Entwicklung und Förderung innovativer Maßnahmen zusammengeschlossen haben.

Die Standortsicherung für seine Mitglieder ist $\mathrm{zu}$ unterstützen, wobei eine wirtschaftliche Vorreiterrolle in Europa anzustreben ist, d. h. eine unangefochtene Rolle der heimischen Lokationen im internationalen Wettstreit zwischen Unternehmen und innerhalb der Konzerne zu erreichen.

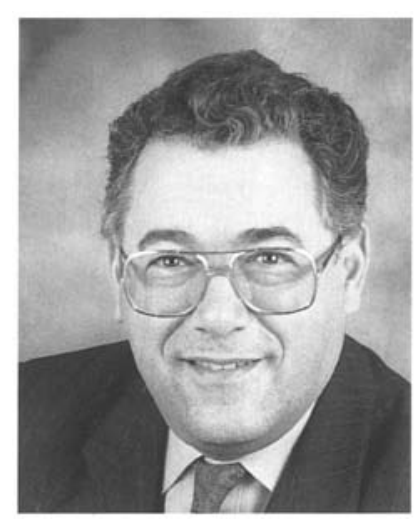

Dipl.-Ing. Helmut Hainitz
Im ersten grundlegenden Schritt war es erforderlich, die eigenen Stärken zu kennen, um sich in den vom "European Rail Research Advisory Council" (ERRAC) genannten fünf strategischen Forschungsprioritäten („Strategic Rail Research Agenda“ - SRRA) entsprechend einbringen zu können. Diese fünf Forschungsprioritäten sind:

- Interoperabilität

- Intelligente Mobilität

- Sicherheit

- Umwelt

- Innovative Werkstoff- und Produktionsmethoden

Möge die vorliegende Publikation als Bestätigung für das vorhandene Innovationspotenzial und als Anregung dienen.

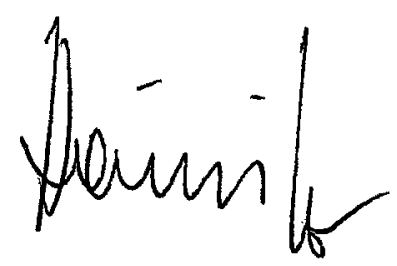

Gen.-Dir.-Stv. i. R. Dipl.-Ing. Helmut Hainitz

Präsident des RTCA - Rail Technology Cluster Austria 